\title{
Impact of p53 loss on reversal and recurrence of conditional Wnt-induced tumorigenesis
}

\author{
Edward J. Gunther, ${ }^{1,2,3,4}$ Susan E. Moody, ${ }^{1,2,3,4}$ George K. Belka, ${ }^{1,2,3,4}$ Kristina T. Hahn, ${ }^{1,2,3,4}$ \\ Nathalie Innocent, ${ }^{1,2,3,4}$ Katherine D. Dugan, ${ }^{1,2,3,4}$ Robert D. Cardiff, ${ }^{5}$ and Lewis A. Chodosh ${ }^{1,2,3,4,6}$ \\ ${ }^{1}$ Department of Cancer Biology, ${ }^{2}$ Department of Cell and Developmental Biology, ${ }^{3}$ Department of Medicine, ${ }^{4}$ Abramson \\ Family Cancer Research Institute, University of Pennsylvania School of Medicine, Philadelphia, Pennsylvania 19104-6160, \\ USA; ${ }^{5}$ Center for Comparative Medicine, University of California, Davis, Davis, California 95616, USA
}

\begin{abstract}
Aberrant activation of Wnt signaling is oncogenic and has been implicated in a variety of human cancers. We have developed a doxycycline-inducible Wnt1 transgenic mouse model to determine the dependence of established mammary adenocarcinomas on continued Wnt signaling. Using this model we show that targeted down-regulation of the Wnt pathway results in the rapid disappearance of essentially all Wnt-initiated invasive primary tumors as well as pulmonary metastases. Tumor regression does not require p53 and occurs even in highly aneuploid tumors. However, despite the dependence of primary mammary tumors and metastases on continued Wnt signaling and the dispensability of p53 for tumor regression, we find that a substantial fraction of tumors progress to a Wnt-independent state and that p53 suppresses this process. Specifically, loss of one p53 allele dramatically facilitates the progression of mammary tumors to a Wnt1-independent state both by impairing the regression of primary tumors following doxycycline withdrawal and by promoting the recurrence of fully regressed tumors in the absence of doxycycline. Thus, although p53 itself is dispensable for tumor regression, it nevertheless plays a critical role in the suppression of tumor recurrence. Our findings demonstrate that although even advanced stages of epithelial malignancy remain dependent upon continued Wnt signaling for maintenance and growth, loss of p53 facilitates tumor escape and the acquisition of oncogene independence.
\end{abstract}

[Keywords: Wnt; p53; mammary gland; inducible transgenic animals]

Received October 18 2002; revised version accepted December 31, 2002.

Wnt signaling during development governs cell proliferation and cell fate in multiple tissues from organisms as diverse as flies and mice (Cadigan and Nusse 1997). Consequently, mutations leading to aberrant activation of Wnt signaling can perturb tissue patterning as well as provide an oncogenic stimulus. Indeed, the Wnt pathway was initially discovered as a result of studies of mammary tumorigenesis in the mouse. Specifically, murine tumors arising as a consequence of infection with the mouse mammary tumor virus (MMTV) were frequently found to harbor retroviral insertions in the vicinity of the Wnt1 locus (formerly known as Int-1) that result in activation of Wnt1 expression (Nusse and Varmus 1982). The subsequent demonstration that forced expression of Wnt1 in the mammary glands of transgenic mice results in hyperplasias and adenocarcinomas of the ductal epi-

${ }^{6}$ Corresponding author.

E-MAIL chodosh@mail.med.upenn.edu; FAX (215) 573-6725.

Article and publication are at http://www.genesdev.org/cgi/doi/10.1101/ gad.1051603. thelium confirmed that Wnt1 is a proto-oncogene (Tsukamoto et al. 1988). A clear link has also emerged between activation of the Wnt pathway and human cancer in that several genes that regulate Wnt signaling have proven to be either proto-oncogenes, as in the case of $\beta$-catenin, or tumor suppressor genes, as in the case of APC and AXIN (Polakis 2000). Consistent with this model, mutations in either $A p c$ or $\beta$-catenin predispose mice to mammary ductal hyperplasias and invasive tumors (Moser et al. 1993; Imbert et al. 2001; Michaelson and Leder 2001; Miyoshi et al. 2002). Although downstream targets of Wnt signaling, such as MYC and cyclin $\mathrm{D} 1$, are known to play important roles in the pathogenesis of human breast cancer, a causal link between aberrant Wnt signaling and human breast cancer is, as yet, unproved. Nevertheless, there is increasing evidence that expression of components of the Wnt signaling pathway itself are altered in a high percentage of human breast cancers (for review, see Bergstein and Brown 1999; Ugolini et al. 2001; Jonsson et al. 2002).

Although it is clear that activation of canonical Wnt signaling plays a role in the genesis of a variety of human 
tumors, whether established tumors remain dependent on continued Wnt signaling for maintenance and growth is unknown (Bienz and Clevers 2000; Taipale and Beachy 2001). While it is possible that Wnt-initiated tumors might be susceptible to antineoplastic strategies involving the targeted down-regulation of Wnt signaling, it is also possible that the accumulation of genetic lesions that foster tumor progression may concomitantly render Wnt pathway activation dispensable for tumor cell survival and proliferation. Unfortunately, analyzing spontaneous events that contribute to tumor progression is difficult, if not impossible, using in vitro systems due to the artificial milieu in which these changes occur. In contrast, genetically engineered mice offer tractable in vivo models that can faithfully recapitulate the stepwise progression of human solid tumors (Siegel et al. 2000). For this reason, inducible transgenic mouse models that permit the conditional expression of oncogenes are of particular interest for modeling the effects of targeted therapies, because the abrogation of oncogene expression following the withdrawal of inducer can simulate drugmediated loss-of-function in a defined oncogenic pathway (Jain et al. 2002). Most notably, the use of a genetic approach to down-regulate oncogenic signaling permits the effects of a targeted therapy to be modeled within an intact tumor composed of multiple cell types in advance of drug development.

To analyze the consequences of down-regulating Wnt signaling in tumors initiated by Wnt pathway activation, we developed a doxycycline-dependent mouse model in which Wnt1 can be conditionally expressed in the mammary epithelium of transgenic mice. Chronic induction of Wnt1 expression in this model results in the stochastic formation of mammary adenocarcinomas over periods of up to a year. In this article we examine the dependence of Wnt1-initiated mammary tumors on continued Wnt signaling in a variety of contexts characteristic of advanced epithelial malignancy. In particular, we tested whether acquisition of a metastatic phenotype, loss of p53 function, or tumor cell aneuploidy preclude tumor regression following abrogation of Wnt1 expression. We demonstrate here that abrogating Wnt1 expression in tumor cells results in the rapid and extensive regression of both primary mammary tumors and pulmonary metastases. These observations provide direct evidence that Wnt pathway down-regulation has potent antineoplastic activity in vivo even against the most advanced stages of epithelial malignancy. In addition, primary tumors lacking p53, including those exhibiting gross aneuploidy, were also found to be capable of undergoing complete regression following the abrogation of Wnt transgene expression. Hence, manifestations of tumor progression such as metastatic spread, chromosomal instability, and loss of p53 do not preclude tumor regression following the targeted down-regulation of an oncogenic signaling pathway. Nevertheless, although our data demonstrate that p53 itself is not required for the regression of Wntinduced tumors, loss of one p53 allele strongly facilitated the emergence of tumor cells that had progressed to a Wnt1-independent state.

\section{Results \\ Doxycycline-inducible mammary-specific expression of Wnt1}

We previously described a transgenic mouse founder line, MTB, that provides mammary-specific expression of the reverse tetracycline transactivator (rtTA) under the control of the MMTV-LTR (long terminal repeat; Gunther et al. 2002). For the work described here, a TetO-Wnt1 responder transgene was constructed carrying a Wnt1 minigene downstream of tet operator sequences. To facilitate transgene detection, this construct encodes a bicistronic mRNA consisting of a luciferase reporter gene translated from an internal ribosome entry site downstream of Wnt1 coding sequences (Fig. 1A). We initially measured doxycycline-dependent luciferase activity in the mammary glands of bitransgenic mice derived by crossing two independent TetO-Wnt 1 founder lines with the MTB transactivator line. This analysis demonstrated that one line, designated TWNT, permitted the tightly regulated, doxycycline-dependent expression of luciferase in the mammary glands of nulliparous bitransgenic MTB/TWNT females (data not shown). This TWNT founder line was used in the experiments described in this report. In addition, the relevant phenotypes observed in MTB/TWNT bitransgenic mice were subsequently confirmed in an independent TetO-Wnt1 founder line.

We tested the extent to which Wnt1 transgene expression could be induced in the mammary glands of MTB/ TWNT bitransgenic mice in response to doxycycline administration. Northern hybridization was performed on total RNA harvested from mammary glands of agematched nulliparous MTB/TWNT females either in the absence of doxycycline treatment or following $4 \mathrm{~d}$ of induction with $2 \mathrm{mg} / \mathrm{mL}$ doxycycline in the drinking water. Wnt 1 transgene expression was readily detected in the mammary glands of doxycycline-treated mice but was undetectable in uninduced mice (Fig. 1B). Consistent with the doxycycline-dependent activation of canonical Wnt signaling, expression of $c-m y c-a$ known transcriptional target of the Wnt pathway-was up-regulated in the mammary glands of MTB/TWNT mice following $4 \mathrm{~d}$ of doxycycline treatment (Fig. 1B; He et al. 1998).

Constitutive Wnt pathway activation in the mammary epithelium of transgenic mice has previously been shown to result in ductal hyperplasia (Tsukamoto et al. 1988). To confirm tight regulation of Wnt pathway activation in MTB/TWNT mice, we examined mammary gland whole-mounts from induced and uninduced MTB/ TWNT females for morphologic alterations. Whereas uninduced MTB/TWNT mice displayed mammary ductal architecture indistinguishable from that of wild-type females, increased ductal side-branching was readily apparent in the mammary glands of MTB/TWNT mice following $96 \mathrm{~h}$ of doxycycline administration (Fig. 1C). On prolonged doxycycline exposure, ductal side-branching increased and was accompanied by precocious lobuloalveolar development. In contrast, the mammary glands of 
A

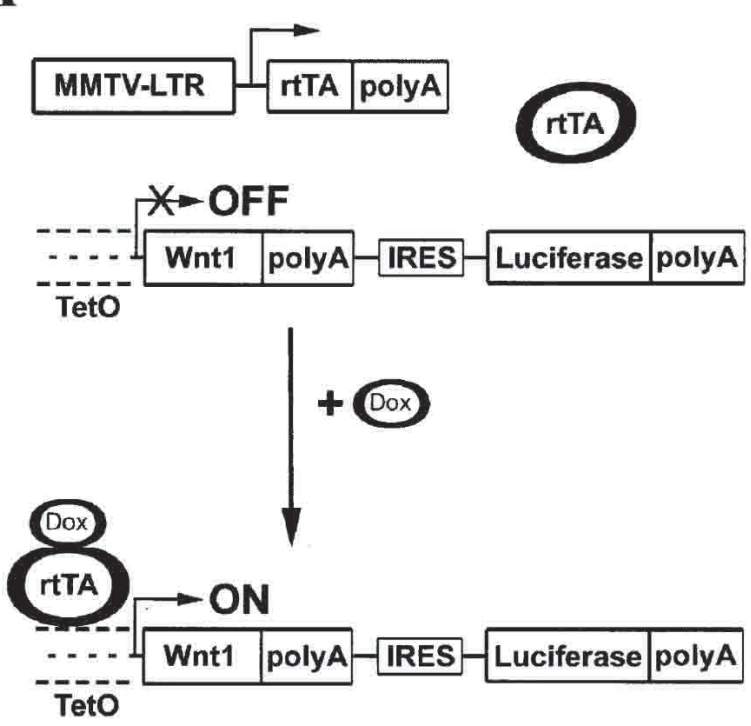

\section{B}

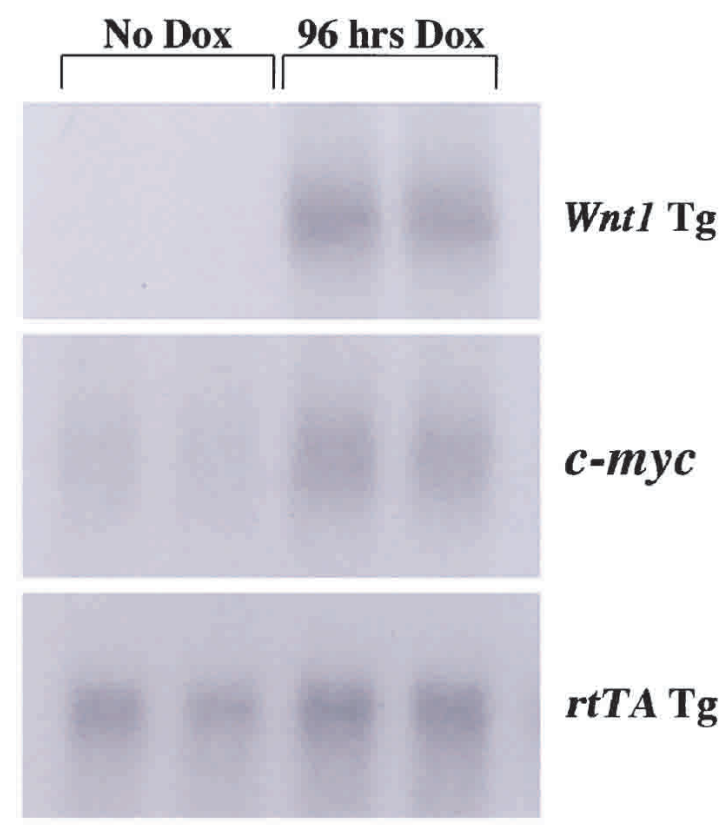

C

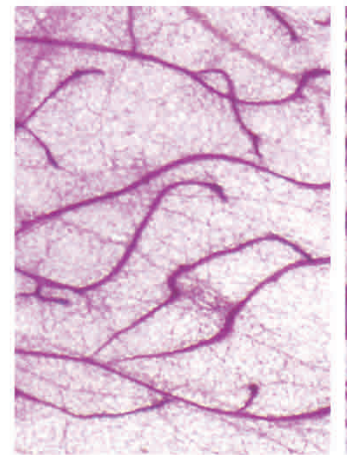

6 wks, No Dox

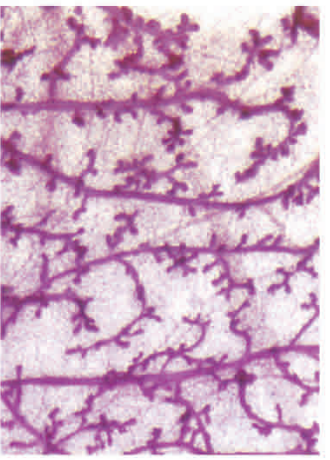

96 hrs Dox

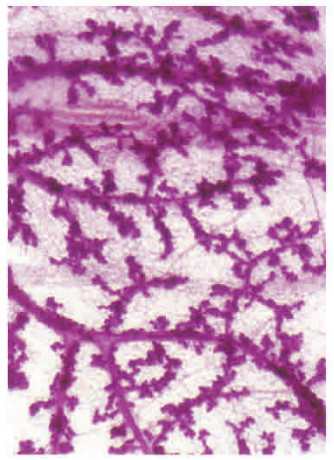

2 wks Dox

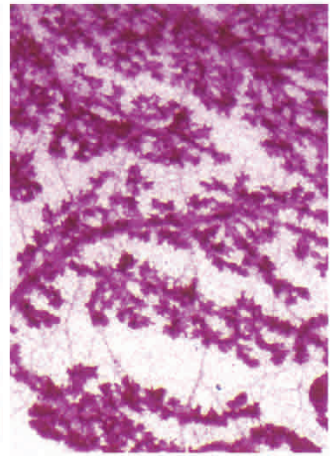

30 wks Dox

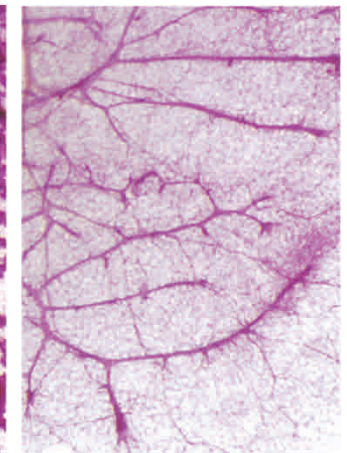

36 wks, No Dox

Figure 1. Doxycycline-dependent activation of Wnt signaling in MTB/TWNT mice. (A) Schematic depicting the strategy to permit doxycycline-dependent expression of Wnt1. Bitransgenic mice carrying both the MMTV-rtTA-pA (MTB) and TetO-Wnt1 (TWNT) transgenes express the rtTA chimeric transcription factor in the mammary epithelium but do not express the bicistronic message encoded by the independently integrated TWNT transgene driven by a minimal CMV promoter containing multimerized tet operator sequences. In the presence of inducer, rtTA binds doxycycline and undergoes a conformational change that facilitates recognition of tet operator sequences and activation of the TWNT promoter. (B) Doxycycline-dependent expression of Wnt1 in the mammary glands of MTB/TWNT mice. Northern hybridization analysis of total RNA from the mammary glands of 5-week-old nulliparous bitransgenic MTB/TWNT female mice. Mice were either left untreated or administered $2 \mathrm{mg} / \mathrm{mL}$ doxycycline in drinking water for $96 \mathrm{~h}$. Note coordinate up-regulation of $c$-myc, a known transcriptional target of canonical Wnt signaling, and the Wnt1 transgene. $(C)$ Doxycycline-dependent mammary ductal side-branching and hyperplasia in MTB/TWNT mice. Mammary glands harvested from MTB/ TWNT and wild-type FVB/N control mice after treatment with doxycycline (Dox) as indicated were spread on slides, fixed, and subjected to carmine staining.

uninduced bitransgenic mice remained indistinguishable from those of age-matched wild-type controls (Fig. $1 \mathrm{C}$; data not shown).

\section{MTB/TWNT mice develop mammary} adenocarcinomas that metastasize to the lung

To determine whether sustained induction of transgene expression would result in mammary tumorigenesis in
MTB/TWNT mice, a cohort of 52 bitransgenic females was treated with doxycycline beginning at 4-6 wk of age. Greater than $90 \%$ of these mice developed tumors in a stochastic manner within $1 \mathrm{yr}$ of starting doxycycline with kinetics $\left(\mathrm{T}_{1 / 2}=140 \mathrm{~d}\right)$ resembling that described for MMTV-Wnt1 mice (Fig. 2A; Li et al. 2000). Similar to the spectrum of tumor histologies described in MMTVWnt1 mice, mammary tumors arising in MTB/TWNT mice predominantly consisted of invasive adenocarcino- 
A

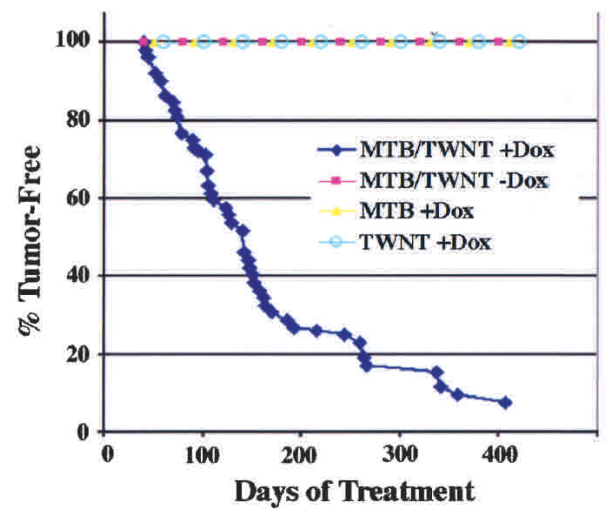

B

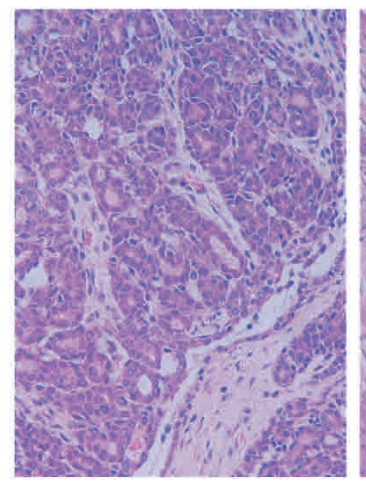

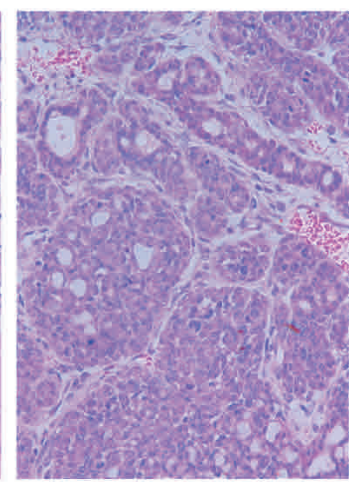

Figure 2. Mammary tumorigenesis in chronically induced MTB/TWNT mice. (A) Tumor incidence curves for transgenic mice. A cohort of 52 female bitransgenic MTB/TWNT mice was administered $2 \mathrm{mg} / \mathrm{mL}$ doxycycline beginning at 4-6 wk of age and monitored twice weekly for tumor formation. Tumors arose with a median latency of $20 \mathrm{wk}$ of doxycycline induction. No tumors arose in control cohorts that included doxycycline-induced monotransgenic mice (MTB, n =20; TWNT, n = 15) and uninduced bitransgenic MTB/ TWNT mice $(\mathrm{n}=9)$. (B) MTB/TWNT mice develop mammary adenocarcinomas. H\&E sections from mammary tumors arising in doxycycline-induced MTB/TWNT mice. The middle panel depicts an area of squamous differentiation seen in a subset of MTB/TWNT tumors.

mas, many of which contained regions of squamous metaplasia (Fig. 2B; Miyoshi et al. 2002). Occasional adenosquamous and squamous cell carcinomas were also noted. Importantly, control cohorts of doxycyclinetreated monotransgenic female mice (MTB, $\mathrm{n}=20$; TWNT, $\mathrm{n}=15$ ) and untreated bitransgenic MTB/TWNT female mice $(\mathrm{n}=9)$ remained tumor-free over periods exceeding 1 yr (Fig. 2A). Thus, tumorigenesis in this model system is highly penetrant and dependent on transgene induction by doxycycline.

Lung metastases are known to arise in a fraction of MMTV-Wnt1 transgenic mice harboring primary mammary tumors (Li et al. 2000). Accordingly, we sacrificed a subset of mammary tumor-bearing MTB/TWNT mice and examined organs at necropsy for evidence of metastatic spread. Gross lung nodules were evident in 3 of 10 mice that had harbored palpable mammary tumors for 4-6 wk prior to sacrifice. Histological analysis of lung lesions confirmed the presence of adenocarcinoma with features characteristic of Wnt1-initiated mammary tumors (Fig. 4A; data not shown).

\section{Wnt1-initiated mammary adenocarcinomas require Wnt signaling for tumor maintenance}

The long tumor latency observed in doxycycline-treated MTB/TWNT mice suggests that additional genetic lesions are required for tumorigenesis. However, whether established tumors that are initiated by aberrant activation of the Wnt pathway remain dependent on continued Wnt signaling for maintenance of the transformed state remains an important question. Consequently, we asked whether down-regulation of Wnt1 expression in Wnt1initiated mammary tumors would result in tumor regression.

Mammary tumors arising in a cohort of doxycycline- treated MTB/TWNT mice were biopsied when they reached a diameter of $10-15 \mathrm{~mm}$, and samples were archived for histologic and molecular analyses. To ensure that surgery had not interfered with tumor growth, animals were maintained on doxycycline for an additional 10-20 d until tumors had grown to at least their prebiopsy dimensions. Doxycycline was then withdrawn and tumor size was monitored as a function of time. Strikingly, nearly all tumors $(33 / 35 ; 94 \%)$ rapidly and fully regressed to a nonpalpable state following doxycycline withdrawal, with a mean time to complete regression of 2 wk (Fig. 3A; Table 1). In contrast, growth of biopsied mammary tumors continued unabated in control MTB/ TWNT mice maintained on doxycycline, necessitating their sacrifice within 4 wk $(n=6)$. Of the two tumors that failed to regress fully following doxycycline withdrawal, one regressed to a $5-\mathrm{mm}$ residual lesion that remained stable in size for several months and was found

Table 1. Tumor regression post-doxycycline withdrawal: p53(+/+) vs. p53(+/-) mice

\begin{tabular}{lrc}
\hline & \multicolumn{2}{c}{ Tumor regression } \\
\cline { 2 - 3 } Genotype & Complete & Incomplete \\
\hline MTB/TWNT/p53(+/+) & $33(94 \%)$ & $2(6 \%)$ \\
MTB/TWNT/p53(+/-) & $18(60 \%)$ & $12(40 \%)$ \\
Tumors with $p 53 \mathrm{LOH}$ & $5(71 \%)$ & $2(29 \%)$ \\
Tumors without $p 53 \mathrm{LOH}$ & $13(57 \%)$ & $10(43 \%)$ \\
\hline
\end{tabular}

Mammary tumors arising in MTB/TWNT/p53(+/+) and MTB/ TWNT/p53(+/-) mice were biopsied, and tumor size was monitored following doxycycline withdrawal. Tumors occurring in p53(+/-) mice were significantly less likely to regress completely following doxycycline withdrawal than tumors occurring in $p 53(+/+)$ mice $(\mathrm{p}<0.005$, chi-square). In addition, tumors that failed to fully regress promptly resumed growth. 
at necropsy to be a cystic lesion containing necrotic debris. The remaining tumor regressed to $3 \mathrm{~mm}$ in size before resuming growth several weeks later. We also observed reversible Wnt1-initiated mammary tumorigenesis using a second TetO-Wnt1 founder line, indicating that the development and regression of tumors in this model does not depend on a particular transgene integration site (data not shown). In aggregate, our findings demonstrate that the vast majority of Wnt1-initiated primary mammary tumors remain dependent on continued Wnt signaling for maintenance of the transformed state.

To analyze the short-term consequences of doxycycline withdrawal on transgene expression and Wnt pathway activation in Wnt1-initiated tumors, freshly harvested fragments of histologically confirmed mammary adenocarcinomas derived from doxycycline-treated MTB/TWNT mice were explanted onto the flanks of syngeneic wild-type $\mathrm{FVB} / \mathrm{N}$ female hosts maintained on doxycycline. Following tumor graft outgrowth on doxycycline, tumor tissue was harvested from deinduced host mice at $0,18,36,54$, and $96 \mathrm{~h}$ post-doxycycline withdrawal, and luciferase activity was measured as an index of transgene expression levels. This analysis revealed that steady-state levels of luciferase activity declined more than 500-fold within $36 \mathrm{~h}$ of doxycycline withdrawal and fell to undetectable levels by $54 \mathrm{~h}$ (Fig. 3B). Northern hybridization confirmed that Wnt1 transgene expression was markedly down-regulated within $18 \mathrm{~h}$ following doxycycline withdrawal, continued to fall at $36 \mathrm{~h}$, and was undetectable by $54 \mathrm{~h}$ (Fig. 3C). Consistent with a decline in canonical Wnt signaling that temporally coincides with the abrogation of Wnt1 transgene expression, Northern analysis further revealed a marked decline in the expression levels of two well described transcriptional targets of the canonical Wnt pathway, $c-m y c$ and Tcf-7, within 36-54 h following doxycycline withdrawal (Fig. 3C; He et al. 1998; Roose et al. 1999).

\section{Lung metastases remain dependent on Wht signaling}

We hypothesized that the genetic alterations required for mammary tumor cells to survive and proliferate at distant sites might also promote Wnt-independent tumor maintenance. Such a model would suggest that metastatic Wnt1-initiated tumor cells would be less dependent on continued Wnt signaling than cells from the primary tumor. To test this hypothesis, metastatic lesions were identified in the lungs of mice bearing solitary mammary tumors and explanted onto the flanks of FVB/N host females maintained on doxycycline. As a paired control, the primary mammary tumor that was the presumed source of each metastasis was explanted onto the contralateral flank of each host mouse. Tumor outgrowths derived from lung lesions and primary mammary gland lesions grew at comparable rates /data not shown) and were histologically indistinguishable from MTB/TWNT primary mammary tumors (Fig. 4A). As above, tumor outgrowths were biopsied when they reached a diameter of $10-15 \mathrm{~mm}$, and host animals were maintained on doxycycline for an additional $2 \mathrm{wk}$ to en- sure continued tumor growth prior to the withdrawal of doxycycline.

Strikingly, the abrogation of transgene expression by doxycycline withdrawal resulted in the rapid and complete regression of grafts derived from both pulmonary metastatic lesions and primary mammary tumors (Fig. 4B). One-hundred percent $(\mathrm{n}=7)$ of established outgrowths from explanted pulmonary lesions derived from three independent MTB/TWNT mice regressed to a nonpalpable state within 2-4 wk of doxycycline withdrawal (Fig. 4B; data not shown). The rates of tumor regression were similar for explants derived from primary tumors and metastases (Fig. 4B). These findings demonstrate that even highly advanced stages of Wnt1-induced malignancy remain dependent upon continued Wnt signaling for maintenance of the transformed state.

\section{Tumor regression does not require p53 and occurs in aneuploid tumors}

Varmus and coworkers have demonstrated that introducing null alleles of p53 into an MMTV-Wnt1 mouse model yields mammary tumors with features characteristic of advanced human malignancy, such as aggressive histology and genetic instability (Donehower et al. 1995, 1996). In light of these results, we wished to determine whether p53 loss-of-function would interfere with tumor regression following the abrogation of Wnt signaling. Following crosses with p53-deficient mice, cohorts of female bitransgenic MTB/TWNT mice that were homozygous, heterozygous, or wild-type for p53 null alleles were chronically induced with doxycycline beginning at 4-6 wk of age until mammary tumors developed. As before, tumors were biopsied and tumor size monitored following doxycycline withdrawal. For MTB/TWNT/p53(-/-) mice, assessment of the dependence of mammary tumors on continued Wnt signaling was hindered by the development of lymphomas and sarcomas, which typically preceded the development of mammary tumors. Therefore, to facilitate completion of the mammary tumor regression protocol, we found that a dose of 5 Gy of ionizing radiation accelerated mammary tumorigenesis while delaying the onset of clinically significant nonmammary tumors (data not shown).

Despite lacking p53, mammary tumors arising in chronically induced MTB/TWNT/p53(-/-) irradiated female mice were capable of regressing to a nonpalpable state when Wnt1 expression was abrogated following doxycycline withdrawal (Fig. 5A). These tumors displayed histologies typical of Wnt1-initiated, invasive adenocarcinomas (data not shown). Analysis of tumor size as a function of time showed that $p 53$-null tumors $(\mathrm{n}=6)$ regressed at a similar rate as tumors arising in p53 wildtype mice (Fig. 5A; data not shown). One mammary tumor arising in an unirradiated MTB/TWNT/p53(-/-) mouse also promptly regressed following doxycycline withdrawal (Fig. 5A, tumor 22134-3R). These results demonstrate that p53 is not required for tumor regression following the down-regulation of Wnt signaling.

We next considered the possibility that the conse- 

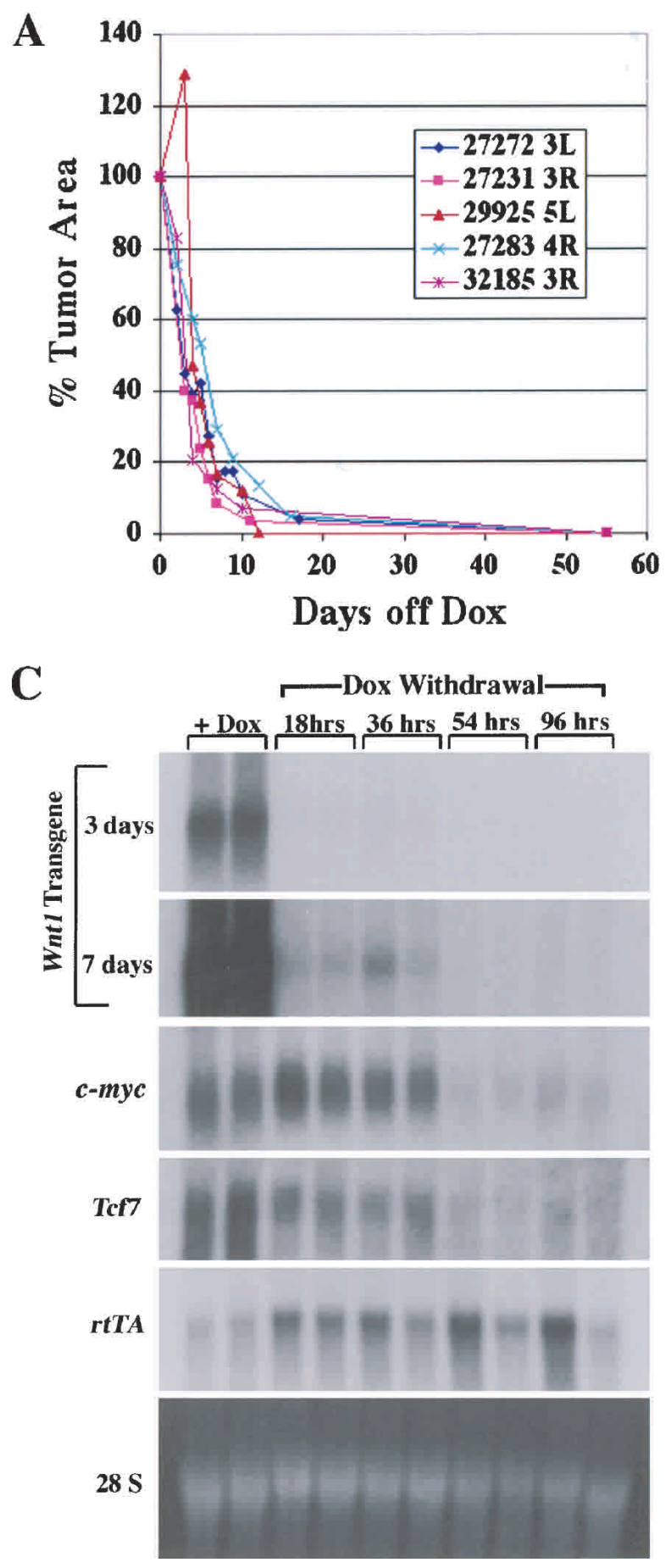

quences of p53 loss-of-function occurring as a somatic event during tumor progression might differ from those due to p53 loss-of-function occurring in the germline. Accordingly, we analyzed tumors arising in MTB/ TWNT/p53(+/-) mice for somatic loss-of-heterozygosity (LOH) events by performing Southern hybridization on tumor-derived DNA. Consistent with previous findings, a subset of tumors (10 out of $51,19.6 \%$ ) exhibited complete or partial LOH at the $p 53$ locus reflecting selective loss of the wild-type p53 allele (Fig. 5B). Because p53
B

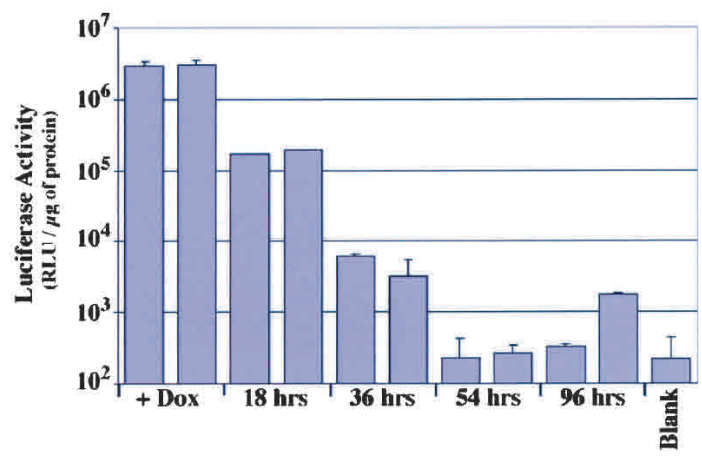

Figure 3. Regression of mammary tumors and downregulation of Wnt signaling following doxycycline withdrawal. (A) Regression of primary mammary tumors in MTB/TWNT mice. The size of biopsy-proven mammary tumors arising in chronically induced MTB/TWNT mice was monitored following doxycycline withdrawal. Shown are representative regression curves for five independent tumors; 33 of $35(94 \%)$ tumors analyzed regressed to a nonpalpable state. $(B)$ Prompt down-regulation of luciferase activity in MTB/TWNT mammary tumors. Mammary tumors were explanted onto the flanks of doxycycline-treated female mice, and tumor outgrowths were harvested from hosts maintained on doxycycline and hosts from whom doxycycline had been withdrawn for the indicated times. A representative analysis performed on grafts derived from a single tumor is shown, with duplicate grafts at each time point. $(C)$ Down-regulation of Wnt signaling in MTB/TWNT mammary tumors. Northern hybridization analysis of total RNA from MTB/TWNT tumor explants. Wnt1 transgene expression is down-regulated within $18 \mathrm{~h}$ (top two panels) but remains detectable until $54 \mathrm{~h}$ (second panel from top, 7-d exposure). Down-regulation of two known transcriptional targets of canonical Wnt signaling, $c-m y c$ and Tcf7, occurs in response to abrogation of Wnt 1 transgene expression. Continued expression of rtTA at 54 and $96 \mathrm{~h}$ post-doxycycline withdrawal demonstrates that downregulation of Wnt1 transgene, c-myc, and Tcf7 is not solely a consequence of loss of tumor cells or tumor cell viability.

loss-of-function was shown to correlate with genomic instability and aneuploidy in mouse mammary tumors (Donehower et al. 1995), we analyzed tumors arising in MTB/TWNT/p53(+/-) mice for DNA content using flow cytometry (Fig. 5C). Although nearly all tumors analyzed that retained the wild-type $p 53$ allele were found to be diploid by FACS analysis (21 of 22 tumors, 95\%), the majority of tumors with detectable $\mathrm{LOH}$ were found to be tetraploid or otherwise aneuploid (8 of 10 tumors, $80 \%$; $p 53-/+$ versus $p 53-/-, \mathrm{p}<0.001)$. In addition, some 
$\mathbf{A}$

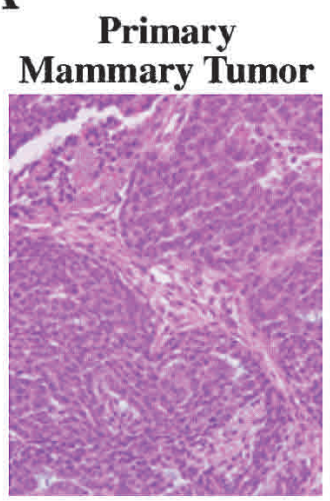

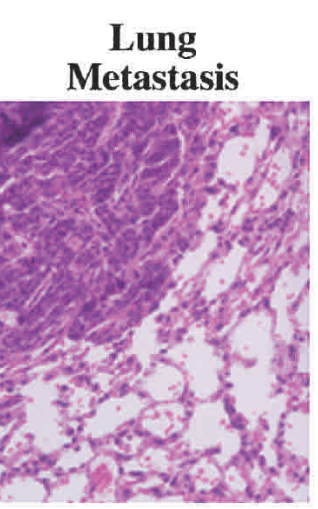

Explanted Metastasis

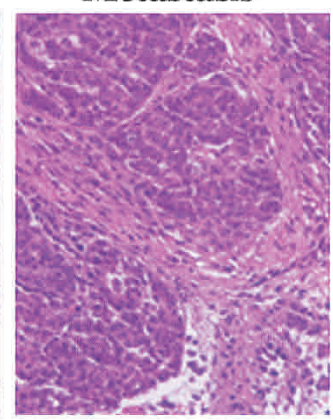

B

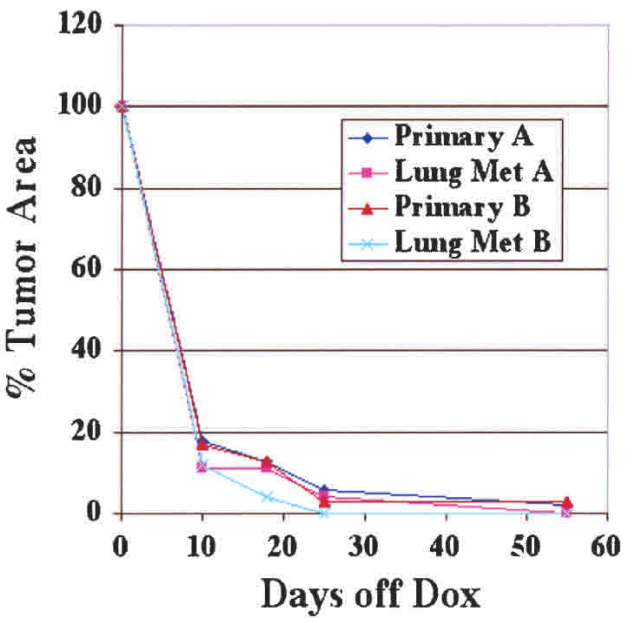

Figure 4. Regression of lung metastases following doxycycline withdrawal. Lung metastases $(\mathrm{n}=7)$ arising in three independent MTB/TWNT mice were explanted onto the flanks of wild-type FVB/N hosts maintained on doxycycline. The primary mammary tumor presumed to be the source of each metastasis was grafted onto the contralateral flank of each host mouse. $(A)$ The histology of H\&E-stained sections derived from lung metastases closely resembled that of primary tumors, both in the case of in situ metastases (middle panel) and explanted metastases (right panel). (B) Similar rate and extent of regression for explants derived from primary mammary tumors and lung metastases. Regression curves for duplicate explants derived from a representative primary-metastasis pair.

tumors displayed evidence of multiple aneuploid cell populations. Thus, consistent with the purported role of p53 in maintaining genomic integrity, aneuploidy was strongly correlated with $p 53 \mathrm{LOH}$ in tumors arising in $\mathrm{MTB} / \mathrm{TWNT} / p 53(+/-)$ mice.

Similar to our findings in mice homozygous for germline deletions in $p 53$, tumors rendered p53-deficient by somatic LOH were also capable of complete regression following doxycycline withdrawal. Notably, this continued dependence on Wnt signaling for tumor maintenance was observed both in diploid tumors and in tumors containing aneuploid cell populations (Table 2). Thus, neither somatically acquired p53 loss-of-function nor the chromosomal instability associated with p53 loss were sufficient to prevent tumor regression following abrogation of Wnt1 expression.

\section{p53 deficiency is associated with incomplete tumor regression}

The chromosomal instability observed in Wnt1-initiated tumors that had undergone somatic loss of p53 function suggested that tumors arising in MTB/TWNT/p53/+/-) mice might be composed of heterogeneous tumor cell populations. We hypothesized that such heterogeneity would increase the frequency with which tumor cells would arise that had acquired the ability to grow in the absence of Wnt1 transgene expression. Consistent with this hypothesis, we observed that incomplete tumor regression was significantly more likely to occur in MTB/ TWNT/p53(+/-) compared to $\mathrm{MTB} / \mathrm{TWNT} / p 53(+/+)$ mice (Table 1; Fig. 5D). Virtually all tumors (33/35 tumors, $94 \%)$ arising in $\mathrm{MTB} / \mathrm{TWNT} / \mathrm{p} 53(+/+)$ mice regressed to a nonpalpable state following doxycycline withdrawal, whereas $40 \%$ of tumors (12/30) arising in MTB/TWNT/p53|+/-) mice failed to regress to a nonpalpable state and subsequently resumed growth in the absence of doxycycline treatment $(\mathrm{p}<0.005$, chi-square). These doxycycline-independent tumors grew aggressively, typically necessitating sacrifice of the host within 2-3 wk. Wht1 transgene expression was undetectable by Northern analysis in four of five doxycycline-independent tumors analyzed, indicating that aberrant activation of transgene expression is not a common mechanism underlying doxycycline-independent tumor growth (data not shown). Thus, although p53 itself is not required for tumor regression following down-regulation of Wnt signaling, loss of a single p53 allele facilitates the emergence of tumor cells that are capable of growth in the absence of Wnt1 transgene expression.

\section{Recurrence of fully regressed mammary tumors is accelerated by 553 deficiency}

To test whether the down-regulation of Wnt signaling in established tumors could lead to a durable tumor response, we monitored a cohort of 34 chronically induced MTB/TWNT/p53(+/+) mice in whom biopsy-documented mammary tumors had arisen during doxycycline treatment and had subsequently regressed to a nonpalpable state following doxycycline withdrawal. Ten tumors $(29 \%)$ occurred in this cohort within 1 yr. Notably, the doxycycline-independent mammary tumors that arose invariably did so in the mammary gland that had previously harbored a Wnt1-initiated tumor, despite the fact that each mouse had initially harbored only a solitary tumor occupying one of 10 mammary glands while on doxycycline. The failure to observe secondary tumors 
A

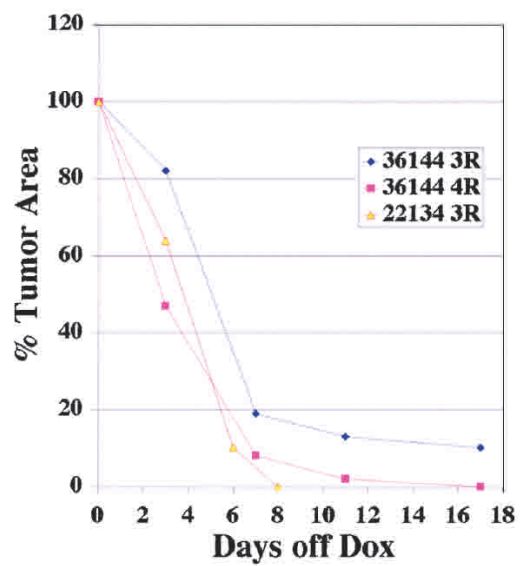

B
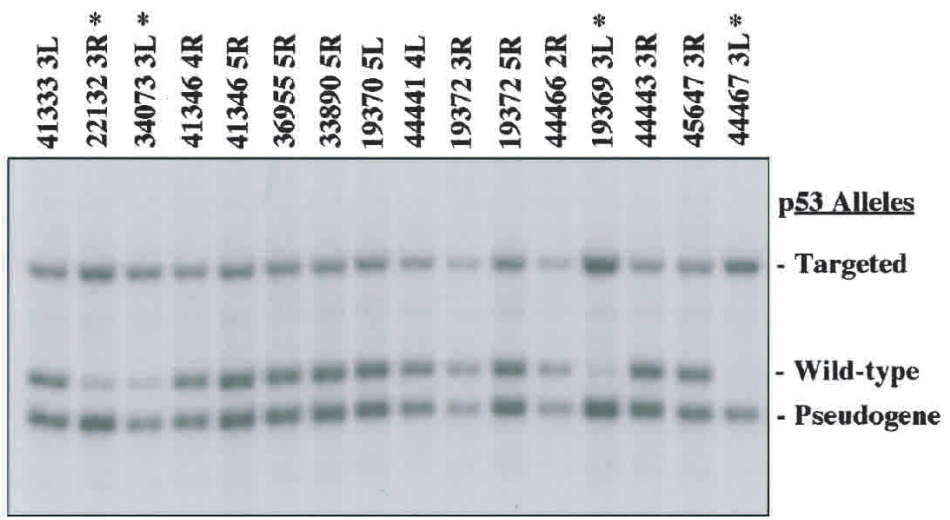

C

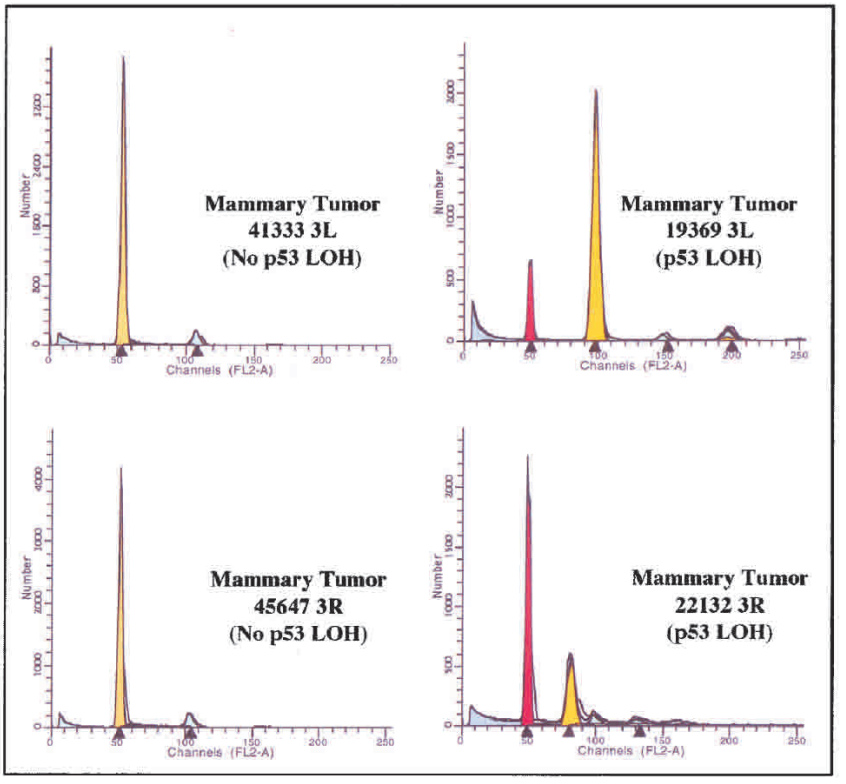

D

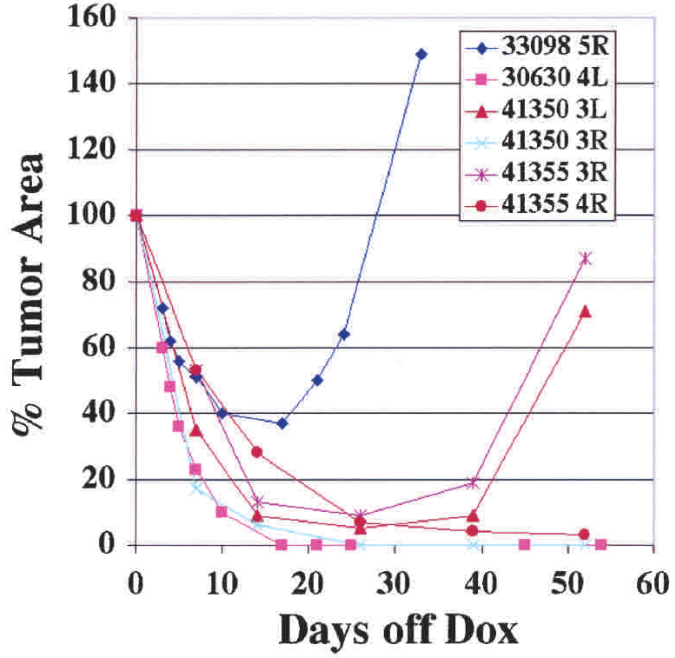

Figure 5. Effect of p53 loss-of-function on the occurrence of tumor aneuploidy and tumor regression following abrogation of Wnt signaling. (A) Regression of MTB/TWNT tumors in the absence of p53. The size of biopsy-proven mammary tumors arising in chronically induced MTB/TWNT/p53(-/-) mice $(\mathrm{n}=7)$ was monitored following doxycycline withdrawal. Regression curves for three representative tumors are shown. Tumors lacking p53 by germline transmission of two null alleles regressed with similar kinetics as tumors arising in mice with wild-type $p 53$ alleles (cf. Figs. 5A and 3D). Tumor recurrence could not be assessed in $p 53(-/-)$ animals due to the rapid occurrence of lymphomas. (B) p53 loss of heterozygosity (LOH) in a subset of tumors arising in MTB/TWNT/p53/+/-) mice. Southern hybridization analysis was performed on genomic DNA derived from mammary tumors arising in mice inheriting a single null allele for $p 53$. Selective loss of the wild-type $p 53$ allele was apparent in a subset of these mammary tumors (lanes marked with $\left.{ }^{\star}\right) .(C)$ Correlation of tumor aneuploidy with $p 53 \mathrm{LOH}$. The ploidy of tumors arising in MTB/TWNT/p53(+/-) mice was determined by flow cytometric analysis of propidium iodide-stained nuclei. Representative DNA histograms for tumors without (left panels) and with (right panels) detectable p53 LOH by Southern analysis are shown. Aneuploidy was common in tumors with detectable $p 53 \mathrm{LOH}$ (6 of 7 tumors, 86\%), but rare in tumors without detectable p53 LOH (1 of 17 tumors, 6\%). (D) Incomplete regression and regrowth of a subset of tumors arising in MTB/TWNT/p53|+/-) mice. The size of biopsy-confirmed mammary tumors arising in $\mathrm{MTB} / \mathrm{TWNT} / p 53(+/-)$ mice was monitored following doxycycline withdrawal as above. Regression curves for six representative tumors are shown. Unlike tumors arising in MTB/TWNT/p53|+/+) mice, which only rarely failed to regress completely following doxycycline withdrawal (2 of 35 tumors, $6 \%$; see Fig. 3D), a substantial fraction of tumors arising in MTB/TWNT/p53(+/-) mice regressed incompletely following doxycycline withdrawal and resumed growth (12 of 30 tumors, 40\%).

in previously uninvolved mammary glands, coupled with the complete absence of primary mammary tumors in uninduced MTB/TWNT mice, strongly suggests that these doxycycline-independent tumors represent true recurrences of Wnt1-initiated tumors, rather than de novo tumor formation in the absence of doxycycline. 
Table 2. Dependence of mammary tumors with p53 LOH on Wnt signaling

\begin{tabular}{lllc}
\hline Tumor & Ploidy & Regression & Recurrence \\
\hline 44467 & Diploid & Incomplete & N/A \\
36121 & Diploid & Complete & No (5 wk) \\
22132 & Aneuploid & Incomplete & N/A \\
36915 & Aneuploid & Complete & No (4 wk) \\
33892 & Aneuploid & Complete & Yes (10 wk) \\
22116 & Aneuploid & Complete & Yes (10 wk) \\
36146 & Aneuploid & Complete & N/A \\
\hline
\end{tabular}

Mammary tumors arising in MTB/TWNT/p53|+/-) mice were biopsied and tumor size was monitored following doxycycline withdrawal. Shown is a subset of tumors for which somatic loss of the wild-type $p 53$ allele was detected by Southern analysis. Ploidy was determined on biopsy samples by analysis of DNA content by flow cytometry. Tumors showing complete regression became nonpalpable over a period of 2-3 wk following doxycycline withdrawal whereas tumors showing incomplete regression remained palpable and resumed growth within $3 \mathrm{wk}$. Mice harboring tumors that had completely regressed following doxycycline withdrawal were monitored for tumor recurrences. The time to development of either a tumor recurrence or an unrelated morbid event sacrifice is shown in parentheses. Tumor 36146 was not monitored for recurrence because a lymphoma necessitated sacrifice of the host mouse shortly after the primary mammary tumor regressed to a nonpalpable state. Tumors displaying both somatic loss of the wild-type p53 allele and aneuploidy were still capable of complete regression. N/A, not applicable.

Our findings suggested that a significant fraction of MTB/TWNT mice in which mammary tumors have re- gressed to a nonpalpable state following the withdrawal of doxycycline harbor residual neoplastic disease. Given the association of $p 53$ mutations with the progression of human cancers (Evan and Vousden 2001), we reasoned that p53 deficiency might foster the emergence of Wnt1independent clones from this putative population of residual neoplastic cells, thereby promoting tumor recurrence in the absence of transgene expression. Because tumor recurrence could not be monitored in p53(-/-) mice due to the rapid occurrence of lymphomas, to test this hypothesis we followed a cohort of MTB/TWNT/ p53(+/-) mice harboring biopsy-documented tumors that had fully regressed following transgene deinduction. When observed off doxycycline for periods of up to $1 \mathrm{yr}$, tumor recurrences were found to accrue significantly faster in p53 heterozygous mice compared to p53 wildtype mice $(\mathrm{p}<0.0001)$ and occurred in a greater fraction of these mice (Fig. 6A). Thus, loss of a single p53 allele contributes not only to incomplete tumor regression following doxycycline withdrawal, but also to increased tumor recurrence in animals whose tumors have fully regressed following transgene deinduction. These findings suggest that in mice harboring fully regressed mammary tumors, p53 deficiency results in decreased disease-free survival by fostering the emergence of Wnt1-independent cells from within a dormant tumor cell population.

Selective pressure favoring loss of a single wild-type p53 allele during Wnt1-initiated mammary tumorigenesis has been demonstrated previously (Donehower et al. 1995, 1996). We considered whether a similar selective pressure would favor $p 53$ loss during the emergence of doxycycline-independent mammary tumors following
A

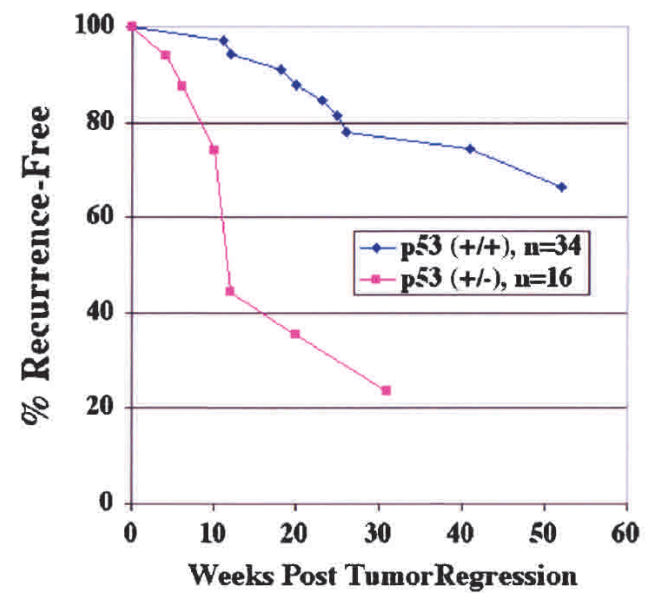

B

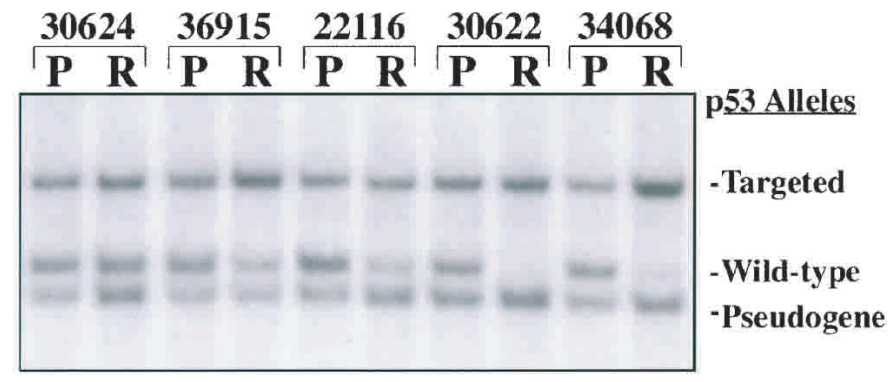

Figure 6. Decreased tumor-free survival in p53 heterozygous mice. (A) Accelerated recurrence of mammary tumors in p53 heterozygous vs. p53 wild-type mice. MTB/TWNT mice that had biopsy-proven mammary tumors that had regressed to a nonpalpable state following doxycycline withdrawal were monitored for the development of doxycycline-independent tumors for periods up to 1 yr. Recurrence of mammary tumors was significantly accelerated in $p 53$ heterozygous vs. $p 53$ wild-type mice $(\mathrm{p}<0.0001, \log$-rank test). (B) p53 LOH in a subset of doxycycline-independent recurrent mammary tumors arising in MTB/TWNT/p53/+/-) mice. Southern hybridization analysis was performed on genomic DNA derived from paired primary mammary tumors $(\mathrm{P})$ and doxycycline-independent recurrent mammary tumors $(\mathrm{R})$ arising in mice inheriting a single null allele for $p 53$. In a subset of primary-recurrence tumor pairs, the recurrent tumor demonstrated selective loss of the wild-type $p 53$ allele. Note that the tumor recurrence could not be assessed in $p 53(-/-)$ mice due to the occurrence of lymphomas. 
abrogation of Wnt1 expression. In fact, a subset of secondary doxycycline-independent mammary tumors arising in MTB/TWNT/p53(+/-) mice showed selective loss of the wild-type p53 allele, even when this allele was retained in the antecedent primary tumor (Fig. 6B). To determine the strength of the association between secondary doxycycline-independent tumors and p53 LOH, 36 tumors consisting of 18 paired primary and secondary tumors arising in MTB/TWNT/p53(+/-) mice were analyzed by Southern blot for loss of the wild-type p53 allele. Eight of the 18 tumor pairs showed selective loss of wild-type p53 exclusively in the secondary doxycyclineindependent tumor (Table 3). Thus, doxycycline-independent mammary tumors were significantly associated with $p 53 \mathrm{LOH}(\mathrm{p}<0.025)$, suggesting that down-regulation of Wnt signaling in Wnt1-initiated mammary tumors leads to selective pressure against a wild-type $p 53$ allele. Alternately, down-regulation of Wnt signaling in Wnt1-initiated tumors may have selected for a subpopulation of cells with an aberrant Wnt pathway.

\section{Discussion}

Mutations that activate canonical Wnt signaling contribute to the pathogenesis of a variety of human malignancies and constitute the earliest and most prevalent genetic abnormalities identified in human colon cancer (Bienz and Clevers 2000; Polakis 2000). Accordingly, drugs capable of the targeted down-regulation of Wnt signaling are currently being developed in the hope that they will possess potent antineoplastic activity. However, whether inhibiting Wnt signaling will prove to be an effective antineoplastic strategy when applied in vivo

Table 3. Selective pressure against wild-type p53 in doxycycline-independent tumors

\begin{tabular}{llrcc}
\hline & & \multicolumn{3}{c}{ p53 status of } \\
& & \multicolumn{3}{c}{ primary tumor } \\
\cline { 3 - 5 } & & No LOH & LOH & Total \\
\hline$p 53$ status of & No LOH & $6(33 \%)$ & $0(0 \%)$ & $6(33 \%)$ \\
doxycycline- & LOH & $8(44 \%)$ & $4(22 \%)$ & $12(67 \%)$ \\
independent & Total & $14(78 \%)$ & $4(22 \%)$ & $18(100 \%)$ \\
secondary & & & & \\
tumor & & & & \\
\hline
\end{tabular}

Primary mammary tumors occurring in doxycycline-treated MTB/TWNT/p53(+/-) mice frequently gave rise to secondary doxycycline-independent tumors following withdrawal of inducer. Shown is a contingency table derived from analysis of 18 primary-secondary tumor pairs. Southern analysis was performed on genomic DNA derived from each tumor to determine whether selective loss of the wild-type p53 allele (LOH) had occurred. Eight of 18 tumor pairs were discordant for p53 LOH, and in all eight discordant pairs, p53 LOH was identified in the secondary tumor. Doxycycline-independent tumors were significantly associated with selective loss of wild-type p53 (p < 0.025, McNemar's test). has heretofore been unknown (Bienz and Clevers 2000; Taipale and Beachy 2001). We addressed this question by developing a novel transgenic mouse model that permits the conditional activation of Wnt signaling in the mammary epithelium. Our data demonstrate that (1) essentially all Wnt-initiated adenocarcinomas arising in wildtype p53 mice remain entirely dependent on continued Wnt signaling for maintenance and growth, (2) lung metastases arising from these tumors are also entirely reversible following the abrogation of Wnt signaling, and (3) p53 is not required for tumor regression in that even aneuploid, p53-deficient tumors are capable of sustained regression following the abrogation of Wnt signaling. These observations provide the first direct evidence that Wnt pathway down-regulation has potent antineoplastic activity when applied against established tumors in vivo, as well as the first demonstration that tumor metastases remain dependent upon an initiating oncogenic event for maintenance of the transformed state. These findings support the development of Wnt pathway inhibitors as anticancer drugs.

However, despite the dependence of Wnt-initiated tumors on continued Wnt signaling and the dispensability of p53 for tumor regression, we find that Wnt-initiated tumors frequently progress to a Wnt-independent state and that $\mathrm{p} 53$ is a critical regulator of this process. Specifically, we observed that (1) a substantial fraction of fully regressed Wnt-initiated tumors spontaneously recur and grow in the absence of transgene expression, (2) tumors arising in $p 53$ heterozygous mice commonly fail to regress fully following abrogation of transgene expression and rapidly resume growth in a Wnt-independent manner, and ( 3 ) even tumors arising in p53 heterozygous mice that do fully regress following transgene deinduction are more likely to recur, and do so at a faster rate than those arising in p53 wild-type mice. Thus, while p53 itself is dispensable for tumor regression, our findings demonstrate that p53 plays an important role in suppressing tumor recurrence.

\section{Reversal of Wnt-initiated tumorigenesis in advanced, genetically complex tumors}

We and others have used inducible transgenic models to demonstrate that primary tumors can remain critically dependent on a single initiating oncogenic stimulus for maintenance and growth (Chin et al. 1999; Felsher and Bishop 1999; Huettner et al. 2000; D'Cruz et al. 2001). We now extend these findings to show that even the most advanced stages of epithelial malignancy, namely metastases, remain similarly oncogene-dependent. Specifically, Wnt1-initiated mammary tumors that have metastasized to the lungs remain dependent on continued Wnt signaling for maintenance of the transformed state. As such, our findings demonstrate that the process of tumor progression culminating in metastasis is not of necessity accompanied by the progression of tumors to a Wnt-independent state. Consequently, our observations suggest that the potential clinical utility of pharmaco- 
logic Wnt pathway inhibitors may extend to the most advanced stages of malignant tumor progression.

In contrast to a number of experimental mouse models of cancer, human cancers are typically genetically complex. For example, human malignancies arising in the setting of an activated Wnt pathway, such as colon cancer, frequently harbor mutations in p53 (Kinzler and Vogelstein 1996). Since p53 is purported to play important roles in apoptosis and the maintenance of genomic stability, it has been suggested that p53 loss might compromise tumor regression following down-regulation of an oncogenic pathway. We found that mammary tumors arising in MTB/TWNT mice carrying two germline $p 53$ null alleles completely regress to a nonpalpable state following doxycycline withdrawal, clearly demonstrating that p53 is not required for the regression of Wnt1-initiated mammary tumors. These results parallel the recent finding that p53 is dispensable for the regression of primary lung tumors initiated by an inducible ras transgene (Fisher et al. 2001). Since mutational inactivation of $p 53$ is normally a somatic event in sporadic human tumors, we went on to characterize the reversibility of Wnt-initiated mammary tumors arising in p53 heterozygous mice that carry an intact p53 allele. Multiple tumors exhibiting selective loss of the wild-type $p 53$ allele were capable of sustained regression following down-regulation of Wnt1. Moreover, consistent with a role for p53 in maintaining genomic integrity, and similar to previous observations (Donehower et al. 1995), the majority of tumors that had undergone loss of the wild-type p53 allele were aneuploid, whereas virtually all tumors retaining the wild-type p53 allele were diploid. Nonetheless, multiple tumors exhibiting both somatic p53 LOH and gross aneuploidy were capable of sustained regression, indicating that neither acquired p53 loss nor acquired chromosomal instability preclude reversal of Wnt-initiated tumorigenesis.

\section{Impaired reversal of Wnt-initiated tumorigenesis in the setting of p53 loss}

Notably, while p53 is clearly dispensable for the complete regression of Wnt1-initiated mammary tumors, loss of one p53 allele dramatically facilitates the emergence of Wnt1-independent tumor cells, both by impairing the regression of primary tumors following doxycycline withdrawal and by promoting the recurrence of fully regressed tumors in animals maintained off doxycycline. Our findings demonstrate that, in addition to the described role for p53 in suppressing the emergence of primary tumors initiated by Wnt1 (Donehower et al. 1995), p53 also plays a role in suppressing the emergence of Wnt1-independent tumors following the abrogation of oncogene expression. Furthermore, our observation that the wild-type $p 53$ allele is selectively lost in a subset of tumors that have acquired the ability to grow in a Wnt1independent manner demonstrates that tumor cell escape from oncogene dependence is accompanied by selective pressure against p53 function in tumor cells. Mutations in $p 53$ have been purported to play a role in tumor resistance to a variety of antineoplastic strategies including cytotoxic chemotherapy, ionizing radiation, and antiangiogenic therapy (Lowe et al. 1993, 1994; Bunz et al. 1999; Johnstone et al. 2002; Yu et al. 2002). Our findings implicate p53 as an important mediator of sustained tumor regression following the targeted downregulation of oncogenic signaling and suggest that, similar to the response of tumors to conventional antineoplastic strategies, the long-term efficacy of targeted therapeutics may be compromised by disruption of the p53 pathway.

\section{Predicting the reversibility of oncogenic pathways}

Unlike our present finding that nearly all Wnt1-initiated mammary tumors remain transgene-dependent, our prior analysis of reversible mammary tumorigenesis initiated by c-MYC indicated that at least $50 \%$ of c-MYCinitiated mammary tumors do not require continued oncogene expression for maintenance of a malignant phenotype (D'Cruz et al. 2001). MYC is a well established transcriptional target of activated Wnt signaling (He et al. 1998). Consistent with this, we found endogenous $c$-myc transcript levels to be coordinately regulated with Wnt1 transgene expression levels in our model. Nevertheless, although c-myc may be a downstream effector of Wnt signaling, the fact that Wnt1-initiated mammary tumors uniformly regress following transgene deinduction, whereas $c-M Y C$-initiated tumors do not, suggests that Wnt1- and $c-M Y C$-initiated mammary tumorigeneses occur via distinct genetic pathways and that mammary tumorigenesis initiated by Wnt pathway activation cannot be wholly attributable to $c$-myc overexpression. These conclusions are consistent with the distinct histologic appearances of Wnt1 and MYC-initiated mammary tumors (Cardiff et al. 1991; Munn et al. 1995). More importantly, the marked differences that we have observed in the oncogene dependence of mammary tumors induced by Wnt1 compared to c-MYC strongly suggest that the probability that a given tumor will become oncogene-independent is fundamentally determined by the oncogenic pathway that initiated it. This conclusion is strengthened by the fact that expression of each oncogene was induced in the mammary epithelium using the same MMTV-rtTA transactivator line in an inbred, genetically identical host background. Accordingly, our observations indicate that the likelihood that an initiating oncogenic pathway will become dispensable for tumor maintenance is an intrinsic function of the specific oncogenic pathway involved.

Drug discovery in the postgenomic era is complicated by the daunting number of potential molecular targets. The prohibitive costs associated with drug development require that potential targets be validated prior to the commitment of resources to a particular therapeutic strategy. Our study provides a paradigm for validating drug targets using inducible transgenic models prior to the development and testing of pharmacologic inhibitors. Here we have shown that this approach permits essentially complete down-regulation of an oncogenic 
stimulus within an intact tumor, thereby simulating the effects of an idealized pharmacologic inhibitor. In addition, such models facilitate the introduction of additional mutations relevant to human cancer as well as the determination, by genetic means, of the molecular pathways required for sustained tumor regression.

Finally, elucidating the specific molecular mechanisms that permit tumors to recur in a transgene-independent manner is an important goal in cancer research, as this should permit the identification of tumor types that are likely or unlikely to respond to the pharmacologic down-regulation of a particular pathway. More importantly, the molecular pathways underlying oncogene resistance may themselves represent fruitful targets against which antineoplastic drugs might be designed. Ultimately, the analysis of transgene-independent tumor recurrences will improve our understanding of the mechanisms by which tumor cells escape from their dependence upon a single oncogenic signaling pathway and will facilitate the development of more effective treatments for this disease.

\section{Materials and methods}

\section{Transgenic mice}

Generation and characterization of the MTB transactivator line has been described (Gunther et al. 2002). To generate the plasmid TMILA, a 2.2-kb cassette containing the internal ribosome entry site from MIGR1 (a gift from Warren Pear, University of Pennsylvania, Philadelphia, PA) followed by the firefly luciferase coding region from pGL3 (Promega) was subcloned upstream of the polyadenylation signal of the plasmid pTet-Splice (GIBCO-BRL). Plasmid TMILA-Wnt1 was generated by subcloning a $1.8-\mathrm{kb}$ fragment containing the murine Wnt1 minigene from plasmid V101 (a gift from R. Nusse, Stanford University, Stanford, CA) downstream of the tet operator sequences in TMILA. The 6.4-kb fragment containing the TetO-Wnt1-IRESLuciferase expression cassette was isolated from vector sequences and prepared for microinjection into fertilized oocytes. Potential founders were identified by screening genomic DNA from tail biopsies for the presence of the transgene using the polymerase chain reaction. All transgenic lines were created and maintained on an inbred FVB/N background. Mice carrying a null allele of $p 53$ generated by gene targeting (Jacks et al. 1994) were obtained from Jackson Laboratories and back-crossed seven generations into an $\mathrm{FVB} / \mathrm{N}$ background prior to crosses with the MTB and TWNT transgenic lines. MTB/TWNT bitransgenic mice carrying null alleles for $p 53$ were typically generated from crosses of $\mathrm{MTB} / p 53(+/-)$ and TWNT/p53(+/-) mice, though $\mathrm{MTB} / p 53(-/-)$ mice were also occasionally used as breeders.

Transgene expression was induced in MTB/TWNT mice by replacing normal drinking water with $5 \%$ sucrose containing 2 $\mathrm{mg} / \mathrm{mL}$ doxycycline, which was changed twice weekly. Mice were inspected for tumors at least twice weekly, and tumors were measured in two dimensions with calipers. Tumor biopsies were performed using isoflurane as an inhalant anesthetic according to IACUC guidelines. Mice treated with ionizing radiation received 5 Gy of total body irradiation from a Cs- 137 source at 3-4 wk of age. Amplification reactions for genotyping animals used the following oligonucleotide pairs: for TWNT, 5'-TGCGGTTCCTGATGTATTTTGC-3' and 5'-TGCATT
CCTTTGGCGAGAGG-3'; for MTB， 5'-TGCCGCCATTAT TACGACAAGC- $3^{\prime}$ and $5^{\prime}$-ACCGTACTCGTCAATTCCAAG GG-3'. Reaction conditions were 40 cycles of $94^{\circ} \mathrm{C}$ for $30 \mathrm{sec}$, $58^{\circ} \mathrm{C}$ for $30 \mathrm{sec}$, and $72^{\circ} \mathrm{C}$ for $30 \mathrm{sec}$.

\section{Northern hybridization}

Total RNA isolation and Northern hybridization were performed as described (Marquis et al. 1995). Blots containing $8 \mu \mathrm{g}$ of total RNA from snap-frozen tumors or mammary tissue were hybridized to cDNA probes containing a 360-bp fragment of rtTA (nucleotide 1441-1800), the entire coding sequence of $W n t 1$, exon 1 of $c-m y c$, or the entire coding sequence of $T c f 7$.

\section{Southern hybridization}

A cDNA probe for exons 7-10 of murine $p 53$ was used to probe Southern blots containing genomic DNA from tumor tissue digested with EcoRI and StuI and passively transferred to GeneScreen Plus nylon membranes (NEN Life Science) as described (Jacks et al. 1994).

\section{Ploidy analysis}

The ploidy of snap-frozen tumor tissue was determined by performing propidium iodide staining of nuclei in hypotonic lysis buffer according to the method of Vindelov (1977); 20,000 events per tumor sample were analyzed on a FACScan flow cytometer. DNA histograms were generated using ModFit LT software (Verity Software House).

\section{Luciferase assay}

Snap-frozen mammary tissue or tumor was homogenized in Passive Lysis Buffer (Promega) using a dounce homogenizer. Homogenates were cleared by centrifugation at $12,000 \mathrm{~g}$, and the supernatant was assayed for protein concentration by the method of Lowry. Luciferase activity was measured using the Dual Luciferase Assay Kit (Promega) and a Monolight 2010 luminometer (Analytical Luminescence Laboratory) according to the manufacturer's instructions.

\section{Morphological analysis}

Mammary gland and tumor samples were fixed in 4\% paraformaldehyde in $1 \mathrm{X}$ phosphate buffered saline overnight and embedded in paraffin. Whole mounts of mammary glands were prepared and stained with carmine alum as described (Gardner et al. 2000). Sections were applied to glass slides and stained with hematoxylin and eosin (H\&E).

\section{Acknowledgments}

We thank Jean Richa and Gary Brown for transgene injections and Nadine Srouji and members of the Chodosh laboratory for helpful discussions and critical reading of the manuscript. This research was supported by NIH grants K08 CA79682 (E.J.G.), CA92190, CA93719, and CA94393 from the National Cancer Institute, U.S. Army Breast Cancer Research Program grant DAMD17-00-1-0401 (S.E.M.), and the University of Pennsylvania Cancer Center Core Support Grant, NCI CA16520.

The publication costs of this article were defrayed in part by payment of page charges. This article must therefore be hereby marked "advertisement" in accordance with 18 USC section 1734 solely to indicate this fact. 


\section{References}

Bergstein, I. and Brown, A.M.C. 1999. WNT genes and breast cancer. In Breast cancer: Molecular genetics, pathogenesis, and therapeutics (ed. A.M. Bowcock), pp. 181-198. Humana Press, Totowa, NJ.

Bienz, M. and Clevers, H. 2000. Linking colorectal cancer to Wnt signaling. Cell 103: 311-320.

Bunz, F., Hwang, P.M., Torrance, C., Waldman, T., Zhang, Y., Dillehay, L., Williams, J., Lengauer, C., Kinzler, K.W., and Vogelstein, B. 1999. Disruption of p53 in human cancer cells alters the responses to therapeutic agents. J. Clin. Invest. 104: 263-269.

Cadigan, K.M. and Nusse, R. 1997. Wnt signaling: A common theme in animal development. Genes \& Dev.11: 3286-3305.

Cardiff, R.D., Sinn, E., Muller, W., and Leder, P. 1991. Transgenic oncogene mice. Tumor phenotype predicts genotype. Am. J. Pathol. 139: 495-501.

Chin, L., Tam, A., Pomerantz, J., Wong, M., Holash, J., Bardeesy, N., Shen, Q., O'Hagan, R., Pantginis, J., Zhou, H., et al. 1999. Essential role for oncogenic Ras in tumour maintenance. Nature 400: 468-472.

D'Cruz, C.M., Gunther, E.J., Boxer, R.B., Hartman, J.L., Sintasath, L., Moody, S.E., Cox, J.D., Ha, S.I., Belka, G.K., Golant, A., et al. 2001. c-MYC induces mammary tumorigenesis by means of a preferred pathway involving spontaneous Kras2 mutations. Nat. Med.7: 235-239.

Donehower, L.A., Godley, L.A., Aldaz, C.M., Pyle, R., Shi, Y.P., Pinkel, D., Gray, J., Bradley, A., Medina, D., and Varmus, H.E. 1995. Deficiency of p53 accelerates mammary tumorigenesis in Wnt-1 transgenic mice and promotes chromosomal instability. Genes \& Dev. 9: 882-895.

Donehower, L.A., Godley, L.A., Aldaz, C.M., Pyle, R., Shi, Y.P., Pinkel, D., Gray, J., Bradley, A., Medina, D., and Varmus, H.E. 1996. The role of p53 loss in genomic instability and tumor progression in a murine mammary cancer model. Prog. Clin. Biol. Res. 395: 1-11.

Evan, G.I. and Vousden, K.H. 2001. Proliferation, cell cycle, and apoptosis in cancer. Nature 411: 342-348.

Felsher, D.W. and Bishop, J.M. 1999. Reversible tumorigenesis by MYC in hematopoietic lineages. Mol. Cell 4: 199-207.

Fisher, G.H., Wellen, S.L., Klimstra, D., Lenczowski, J.M., Tichelaar, J.W., Lizak, M.J., Whitsett, J.A., Koretsky, A., and Varmus, H.E. 2001. Induction and apoptotic regression of lung adenocarcinomas by regulation of a K-Ras transgene in the presence and absence of tumor suppressor genes. Genes \& Dev. 15: 3249-3262.

Gardner, H.P., Belka, G.K., Wertheim, G.B., Hartman, J.L., Ha, S.I., Gimotty, P.A., Marquis, S.T., and Chodosh, L.A. 2000. Developmental role of the SNF1-related kinase Hunk in pregnancy-induced changes in the mammary gland. Development 127: 4493-4509.

Gorre, M.E. and Sawyers, C.L. 2002. Molecular mechanisms of resistance to STI571 in chronic myeloid leukemia. Curr. Opin. Hematol. 9: 303-307.

Gunther, E.J., Belka, G.K., Wertheim, G.B., Wang, J., Hartman, J.L., Boxer, R.B., and Chodosh, L.A. 2002. A novel doxycycline-inducible system for the transgenic analysis of mammary gland biology. FASEB J. 16: 283-292.

He, T.C., Sparks, A.B., Rago, C., Hermeking, H., Zawel, L., da Costa, L.T., Morin, P.J., Vogelstein, B., and Kinzler, K.W. 1998. Identification of c-MYC as a target of the APC pathway. Science 281: 1509-1512.

Huettner, C.S., Zhang, P., Van Etten, R.A., and Tenen, D.G. 2000. Reversibility of acute B-cell leukemia induced by BCRABL1. Nat. Genet. 24: 57-60.
Imbert, A., Eelkema, R., Jordan, S., Feiner, H., and Cowin, P. 2001. Delta N89 $\beta$-catenin induces precocious development, differentiation, and neoplasia in mammary gland. J. Cell Biol. 153: 555-568.

Jacks, T., Remington, L., Williams, B.O., Schmitt, E.M., Halachmi, S., Bronson, R.T., and Weinberg, R.A. 1994. Tumor spectrum analysis in p53-mutant mice. Curr. Biol. 4: $1-7$.

Jain, M., Arvanitis, C., Chu, K., Dewey, W., Leonhardt, E. Trinh, M., Sundberg, C.D., Bishop, J.M., and Felsher, D.W. 2002. Sustained loss of a neoplastic phenotype by brief inactivation of MYC. Science 297: 102-104.

Johnstone, R.W., Ruefli, A.A., and Lowe, S.W. 2002. Apoptosis: A link between cancer genetics and chemotherapy. Cell 108: $153-164$.

Jonsson, M., Dejmek, J., Bendahl, P.-O., and Andersson, T. 2002. Loss of Wnt-5a protein is associated with early relapse in invasive ductal breast carcinomas. Cancer Res. 62: 409-416.

Kinzler, K.W. and Vogelstein, B. 1996. Lessons from hereditary colorectal cancer. Cell 87: 159-170.

Li, Y., Hively, W.P., and Varmus, H.E. 2000. Use of MMTVWnt-1 transgenic mice for studying the genetic basis of breast cancer. Oncogene 19: 1002-1009.

Lowe, S.W., Schmitt, E.M., Smith, S.W., Osborne, B.A., and Jacks, T. 1993. p53 is required for radiation-induced apoptosis in mouse thymocytes. Nature 362: 847-849.

Lowe, S.W., Bodis, S., McClatchey, A., Remington, L., Ruley, H.E., Fisher, D.E., Housman, D.E., and Jacks, T. 1994. p53 status and the efficacy of cancer therapy in vivo. Science 266: $807-810$.

Marquis, S.T., Rajan, J.V., Wynshaw-Boris, A., Xu, J., Yin, G.Y., Abel, K.J., Weber, B.L., and Chodosh, L.A. 1995. The developmental pattern of Brcal expression implies a role in differentiation of the breast and other tissues. Nat. Genet. 11: $17-26$.

Michaelson, J.S. and Leder, P. 2001. $\beta$-catenin is a downstream effector of Wnt-mediated tumorigenesis in the mammary gland. Oncogene 20: 5093-5099.

Miyoshi, K., Shillingford, J.M., Le Provost, F., Gounari, F., Bronson, R., von Boehmer, H., Taketo, M.M., Cardiff, R.D., Hennighausen, L., and Khazaie, K. 2002. Activation of $\beta$-catenin signaling in differentiated mammary secretory cells induces transdifferentiation into epidermis and squamous metaplasias. Proc. Nat1. Acad. Sci.99: 219-224.

Moser, A.R., Mattes, E.M., Dove, W.F., Lindstrom, M.J., Haag, J.D., and Gould, M.N. 1993. ApcMin, a mutation in the murine Apc gene, predisposes to mammary carcinomas and focal alveolar hyperplasias. Proc. Nat1. Acad. Sci.90: 89778981.

Munn, R.J., Webster, M., Muller, W.J., and Cardiff, R.D. 1995. Histopathology of transgenic mouse mammary tumors (a short atlas). Semin. Cancer Bio. 6: 153-158.

Nusse, R. and Varmus, H.E. 1982. Many tumors induced by the mouse mammary tumor virus contain a provirus integrated in the same region of the host genome. Cell 31: 99-109.

Polakis, P. 2000. Wnt signaling and cancer. Genes \& Dev. 14: $1837-1851$.

Roose, J., Huls, G., van Beest, M., Moerer, P., van der Horn, K., Goldschmeding, R., Logtenberg, T., and Clevers, H. 1999 Synergy between tumor suppressor APC and the $\beta$-cateninTcf4 target Tcf1. Science 285: 1923-1926.

Siegel, P.M., Hardy, W.R., and Muller, W.J. 2000. Mammary gland neoplasia: Insights from transgenic mouse models. Bioessays 22: 554-563.

Taipale, J. and Beachy, P.A. 2001. The Hedgehog and Wnt signalling pathways in cancer. Nature 411: 349-354. 
Tsukamoto, A.S., Grosschedl, R., Guzman, R.C., Parslow, T., and Varmus, H.E. 1988. Expression of the int-1 gene in transgenic mice is associated with mammary gland hyperplasia and adenocarcinomas in male and female mice. Cell 55: 619625.

Ugolini, F., Charafe-Jauffret, E., Bardou, V.-J., Geneix, J., Adelaide, J., Labat-Moleur, F., Penault-Llorca, F., Longy, M., Jacquemier, J., Birnbaum, D., et al. 2001. Wnt pathway and mammary carcinogenesis: Loss of expression of candidate tumor suppressor gene SFRP1 in most invasive carcinomas except of the medullary type. Oncogene 20: 58105817.

Vindelov, L.L. 1977. Flow microfluorometric analysis of nuclear DNA in cells from solid tumors and cell suspensions. A new method for rapid isolation and straining of nuclei. Virchows Arch. B Cell Pathol. 24: 227-242.

Yu, J.L., Rak, J.W., Coomber, B.L., Hicklin, D.J., and Kerbel, R.S. 2002. Effect of p53 status on tumor response to antiangiogenic therapy. Science 295: 1526-1528. 


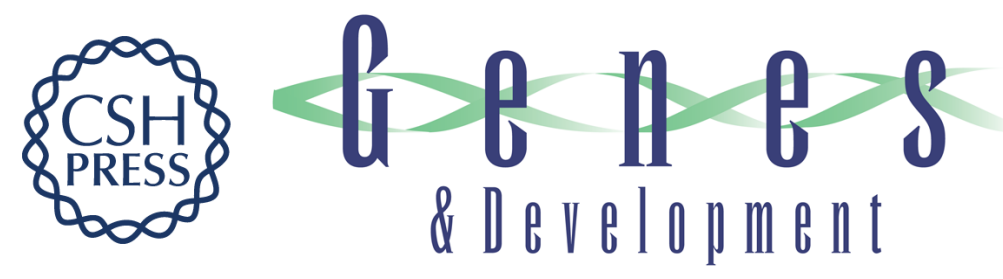

\section{Impact of p53 loss on reversal and recurrence of conditional Wnt-induced tumorigenesis}

Edward J. Gunther, Susan E. Moody, George K. Belka, et al.

Genes Dev. 2003, 17:

Access the most recent version at doi:10.1101/gad.1051603

References This article cites 38 articles, 14 of which can be accessed free at: http://genesdev.cshlp.org/content/17/4/488.full.htmI\#ref-list-1

License

Email Alerting

Receive free email alerts when new articles cite this article - sign up in the box at the top Service right corner of the article or click here.

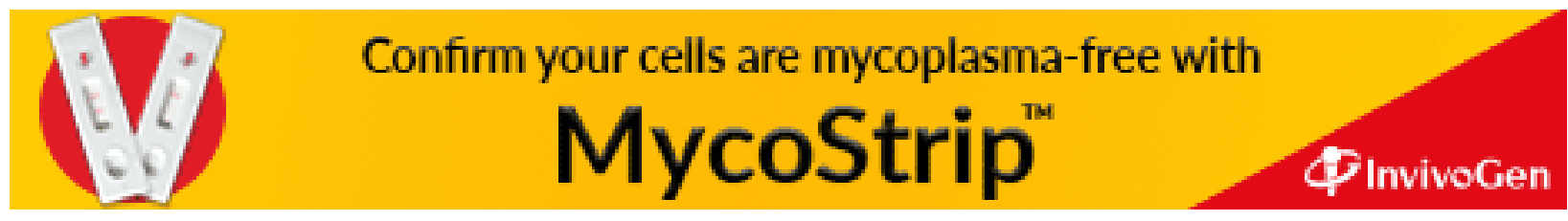

